\title{
Social Presence and Dishonesty in Retail
}

\author{
Susan Siebenaler \\ Abertay University \\ Bell Street, Dundee DD1 1HG, UK \\ susan.siebenaler@gmail.com
}

\author{
Graham I. Johnson \\ NCR Corporation \\ 3 Fulton Road, Dundee DD2 4SW, UK \\ graham.johnson@ncr.com
}

\author{
Andrea Szymkowiak \\ Abertay University \\ Bell Street, Dundee DD1 HG, UK \\ a.szymkowiak@abertay.ac.uk \\ Jan Law \\ Abertay University \\ Bell Street, Dundee DD1 1HG, UK \\ j.law@abertay.ac.uk
}

\author{
Paul Robertson \\ Abertay University \\ Bell Street, Dundee DD1 HG, UK \\ p.robertson@abertay.ac.uk
}

\author{
Kenneth Fee \\ Abertay University \\ Bell Street, Dundee DD1 1HG, UK \\ k.fee@abertay.ac.uk
}

\begin{abstract}
Self-service checkouts (SCOs) in retail can benefit consumers and retailers, providing control and autonomy to shoppers independent from staff, together with reduced queuing times. Recent research indicates that the absence of staff may provide the opportunity for consumers to behave dishonestly, consistent with a perceived lack of social presence. This study examined whether a social presence in the form of various instantiations of embodied, visual, humanlike SCO interface agents had an effect on opportunistic user behaviour, i.e. an individual taking unwarranted advantages. Using a simulated SCO scenario, participants experienced various dilemmas in which they could financially benefit themselves undeservedly. We hypothesised that a humanlike social presence integrated within the checkout screen would receive more attention and result in fewer instances of dishonesty compared to a less humanlike agent. This was partially supported by the results. The findings contribute to the theoretical framework in social presence research. We concluded that companies adopting self-service technology may consider the implementation of social presence in technology applications to support ethical consumer behaviour, but that more research is required to explore the mixed findings in the current study.
\end{abstract}

Embodied agents, anthropomorphism, retail shrinkage, social presence, self-service, eye tracking.

\section{INTRODUCTION}

Opportunistic behaviour has been of interest to researchers of deviant consumer behaviour (Wirtz and McColl-Kennedy, 2010), and is concerned with ways in which consumers choose to take advantage of opportunities during an interaction with a retailer to benefit themselves. While there is substantial research on opportunistic behaviour in traditional staffed retail outlets, research on opportunistic behaviour at self-service checkout (SCO) systems has only recently seen more interest (Beck, 2015; Taylor, 2016).

The use of SCOs (see Figure 1.1 for an example) typically implies that consumers scan, bag and pay for their shopping items independently from staff, who are often deployed elsewhere for customer assistance. Thus, SCO transactions may be associated with reduced physical and/or social presence, the latter being defined here as the perceived presence of others in technology mediated environments (see Short et al., 1976). This type of consumer transaction thus provides an opportunity to investigate the effects of social presence in technological form on dis-/honest consumer behaviour, which was the aim of this study.

In the following we will review the theoretical framework surrounding crime, with a particular focus on how social presence affects human behaviour. We will also discuss definitions of social presence, and how it has been instantiated in retail to reduce theft, followed by a description of the current study.

\subsection{Factors surrounding dishonest behaviour}

The model of the offender as a decision maker underlies much criminological research of deviance by psychologists, economists, and sociologists (Clarke and Cornish, 1985). The concept of "choice structuring properties" (Cornish and Clarke 1987, p.933), which refers to the constellation of opportunities, costs, and benefits attached to particular kinds of crime, can provide a useful framework for analysing crime control policies. According to the Crime Triangle (Clarke and Eck, 2014), occurrence of crime is associated with three factors: 1) a target with opportunity available, 2) ability to obtain a product in a 
specific place, and 3) the offender's desire to complete the crime. Addressing any of these factors can reduce or eliminate the occurrence of crime; for example, opportunity for theft in retail can be counteracted using security tags and surveillance by staff.

In line with this framework, the implementation of surveillance has increased over recent decades (Welsh et al., 2010). Formal surveillance, such as closed-circuit television (CCTV), store staff, security guards, and informal surveillance, such as lighting and placing of products, are seen to be effective measures against crime (Kajalo and Lindblom, 2010). Irrespective of transaction format, however, shrinkage due to customers has been and continues to be an ongoing problem for retailers, with exact figures unlikely to be known. Figures on customer theft vary by year and country (Bamfield, 2004, Baumer and Rosenbaum, 1984, Beck, 2011). Estimates (Beck, 2011) suggest that customer theft accounts for $35 \%$ of all shrinkage, with the majority due to other factors, such as internal theft $(33 \%)$, process failures and inter-company fraud (32\%). While surveillance is generally effective in deterring theft, the use of CCTV has become less effective, as customers have become inured to traditional CCTV (Beck and Willis, 1999). This finding raises the question of whether social presence (i.e. perceived presence of others) can be implemented in other, additional ways to deter crime.

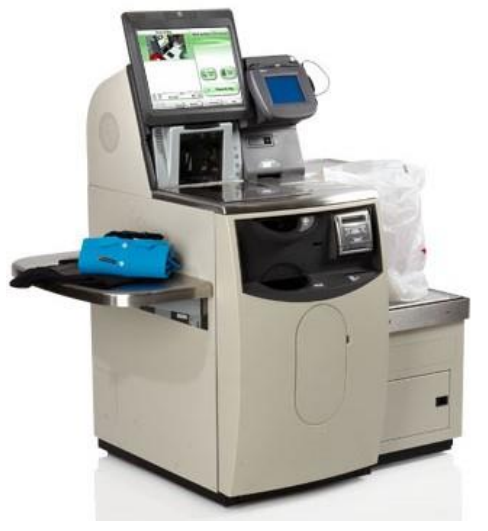

Figure 1.1: Example of a self-service checkout (SCO)

\subsection{Self-service and dishonest behaviour}

In recent years a number of studies has investigated the occurrence of customer theft at self-service (e.g. Beck and Hopkins, 2017), but many companies do not share their findings (Beck, 2011; Beck and Hopkins, 2017). While a recent UK Home Office Report (2015) stated that stores that adopt selfservice are more likely to experience theft than those that do not (Home Office, 2015) a direct link between theft and self-service was not drawn. Stores that do adopt self-service are typically different from those which do not, e.g. are larger, and the report concluded that it cannot be suggested that the use of self-service causes higher levels of shoplifting (Home Office, 2015).

Nevertheless, with the implementation of new technology, new (dishonest) behaviours may arise. For example, customers annoyed by failures during a transaction can blame the machine as a means for justifying theft, which Beck (2011) described as the 'self-scan defence'. Consistent with this notion, Taylor (2016) identified different strategies customers utilize to engage in theft purposefully, but she also reported that frustration may induce people to behave dishonestly, which was likewise described by others (Siebenaler et al., 2017).

Whatever the motivations customers may have, and given that shoplifters themselves perceive formal surveillance as one of the biggest deterrents for stealing (Carmel-Gilfilen, 2013), there may be scope to address dishonest behaviour at SCO via social presence. We propose that the incorporation of social presence within self-service transactions may provide a way to address theft at SCO as the perception of being watched, i.e. a form of social presence, induces people to adopt pro-social, behaviours (e.g. Pfattheicher and Keller, 2015).

Next we will discuss the concept of social presence. Note that the effects of instantiations of social presence in this study is aimed to address typical customer behaviour that may or may not become dishonest during the transaction.

\subsection{Social presence, social agents and proso- cial behaviour}

Short et al. (1976) first introduced the notion of social presence as the salience of others and their interpersonal interactions in mediated communications. Several definitions of social presence have been put forward since, however, they have in common the indication that there is a perception of a shared environment with others (Biocca et al. 2001; Walther, 1992). For example, Romano et al. (2005) stated that a social presence creates the illusion in the mind of the perceiver that another intelligence exists in the environment. Biocca et al. (2003) described social presence as a sense of being with another and the "other" can be human or an artificial intelligence. Co-presence is the dimension of social presence relating to the degree to which an observer believes $s /$ he is not alone (Lee et al. 2016), which may result in an observer modifying his/her behaviour (Zhao, 2003).

Dautenhahn and Billard (2002) proposed that high levels of social presence create feelings of companionship in a user. Baumeister (1982) suggested that the presence of others can lead individuals to alter their behaviour in a manner that communicates a positive self-impression. Consistent with this notion, though while not explicitly studying social presence, Bateson et al. (2006) investigated the effects that 
images of humanlike features have on prosocial behaviour. This research monitored money contributions in an honesty box within a university coffee room. During the collection period, various images were placed next to the honesty box, showing either a pair of eyes (high social presence) or a bunch of flowers (low/no social presence). The findings showed that a pair of eyes resulted in three times more money in the box compared to when the image was of a bunch of flowers. This finding suggests that people were more likely to behave honestly when they experienced a social presence (the image of a pair of eyes) even when only an implied human presence was present.

Research that shows a social presence can be induced by images has also been conducted by Nowak and Biocca (2003), who looked at whether people will respond socially to computer-controlled entities that involved humanlike features, such as eyes. Their findings suggest that people do respond socially to artificial computer agents; in addition, human features designed within computer agents created feelings of a social presence when participants interacted with them. Other work reported that human qualities integrated within an on-screen agent increased user performance in a decision-making task (Shinozawa, 2005).

Sproull et al. (1996) showed that people presented themselves more positively when the interface they interacted with contained a face compared to a textdisplay. In addition, research has highlighted that people will cooperate more, and respond socially to humanlike virtual agents compared to non-human virtual agents, i.e. a dog, and that increasing levels of agent realism can positively influence cooperation (Parise et al., 1999).

Within e-commerce, social presence was found to promote feelings of user trust (Gefen and Straub, 2004) which may then increase customer loyalty (Cyr et al. 2007).

\subsection{Cues to social presence}

Humanlike features have been suggested as being advantageous to human-computer interactions to imply that the computer has particular social skills. Human forms indicate human qualities that evoke perceptions of lifelikeness in the system (Küster et al. 2015). Anthropomorphic representations, i.e. humanlike appearance or behaviour, make the computer appear more intelligent, engaging and capable of higher agency than those with nonhuman visual forms (King and Ohya, 1996; Koda and Maes, 1996). Social interactions between computer agents and humans have been suggested as being similar as humans have a need to care for others (Sproull et al., 1996). In addition, humanlike interfaces induce users to apply impressionformation and management techniques similar to those that would be expected in human-to-human communication (Küster, et al., 2015).

Why are humanlike features, in particular, eyes, so relevant for human behaviour? It has been suggested that humans have involuntary perceptual systems that respond to stimuli of faces and eyes (Emery, 2000; Haxby, et al., 2000). Haxby et al. (2000) state that humans rely on face and eye cues to make social decisions and determine whether or not an individual is trustworthy. Gaze detection has been closely linked with theory of mind, as suggested by Baron-Cohen (1997), in that individuals may perceive $a(n)$ (human) agent's level of interest in him/her via visual behaviours displayed. Gaze behaviour may influence the perception of interest from the agent on the user, also known as the mutual attention mechanism (Peters, 2005). Consistent with mutual attention, users will follow a virtual agent's gaze (Martinez et al., 2011), akin to social gaze in human-human interaction.

\subsection{Experimental rationale and hypotheses}

Based on the previous research, it is reasonable to suggest that virtual characters, especially when they display human physiognomy, more specifically, eyes, may be related to social presence. The question then arises whether different instantiations of 'humanness', and thereby social presence, affect dishonest behaviour in a similar fashion, that is, whether interacting with more humanlike agents results in more honest (prosocial) behaviour compared to less humanlike agents.

To address this, it is essential that agents are seen. Asking observers directly whether they have seen specific elements after they have used an interface may not yield valid answers, as participants may not recall them. For example, Payne et al. (2011) reported that the presence of a visual agent on a simulated SCO scenario induced fewer people to make mistakes compared to a control condition; however, many participants did not recall having seen the agent within the self-service interface. A more reliable procedure may be the recording of where a viewer's gaze is directed on a SCO screen.

Eye tracking technology has been used to capture human gaze behaviour and associated cognitive processes for decades (for a review see Duchowski, 2002), and provides a useful technique to investigate user interactions. Fixation frequency (i.e. how often an observer's gaze dwells in a particular area of interest) is a measure of an area's importance, with more frequent fixations indicating higher importance (Duchowski, 2002). Fixation duration, i.e. how long an observer fixates a particular area of interest, is associated with attention allocation (Henderson, 2007) and durations will be longer on more informative objects than less informative objects (Antes, 1974; Friedman and Liebelt, 1981; Unema et al., 2005). To the extent that an agent is 
perceived to be important, it should attract longer fixations.

This study examined the effects of varying social presence in the form of virtual embodied agents on consumer behaviour in a simulated SCO scenario. Social presence was manipulated by varying the 'humanness' features of onscreen agents as these features generate social presence (Nowak and Biocca, 2003). All agent images contained eyes as human features, as eyes are associated with more prosocial behaviour (Bateson et al., 2006). In addition, realistic looking agents (Parise et al., 1999) elicit more cooperation than non-human (animal) agents. Thus, we pitched a realistic looking agent against two agents that hinted at humanness: one was logo-like, the other cartoon-like. We predicted that the more humanlike agent would result in fewer instances of dishonest behaviour.

To control whether the agents were seen by the user and could exert their impact on the observer, an eye tracker was used to capture gaze behaviour. We also wanted to explore the notion of whether a more humanlike agent may be looked at more, as human faces appear to draw more attention than objects (Devue et al., 2009).

\section{Hypothesis 1 (H1):}

Agents that are more humanlike (higher social presence) result in higher levels of attention than agents that are less humanlike.

\section{Hypothesis 2 (H2):}

Agents that are more humanlike (higher social presence) result in lower levels of dishonest behaviour than agents that are less humanlike.

\section{METHOD}

\subsection{Participants}

Forty-eight participants (25 female, 23 male) with an average age of 24 took part in the research employing a between-subject design for the three levels of social presence manipulation. There were 16 participants each in the human agent condition (HA, highest level of social presence), the cartoon-like agent (CA, medium level of social presence) condition and the logo-like agent condition (LA, lowest level of social presence). All participants were students recruited from Abertay University and reported high levels of experience with computers and self-service checkouts, where they felt confident in using both independently to complete tasks.

\subsection{Set-up of agents and scenarios}

Social presence was varied via the implementation of three levels of humanlike interface agents (Fig- ure 2.1) on a simulated SCO. The least humanlike (low social presence) agent was represented by a logo-like agent (LA) with eyes. The next level was a cartoon-like agent (CA) that resembled a human, with stylised eyes and torso; the highest level of humanness was represented by a female humanlike agent (HA) with shoulders, and associated with the highest social presence condition.

\section{LA - logo-like agent}

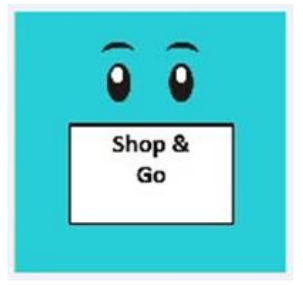

CA - cartoon-like agent

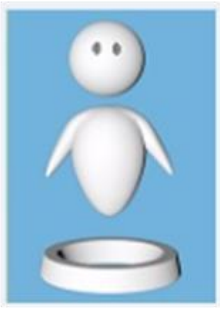

HA - humanlike agent

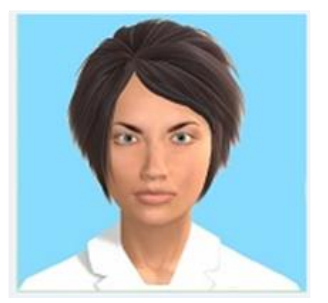

Figure 2.1: Agent images in order of increasing humanlike features from top to bottom panels, with the logo-like agent (LA) shown in the top panel, cartoon-like agent (CA) shown in the middle panel and the humanlike agent (HA) shown in the bottom panel.

A simulated SCO was used to measure participant behaviour (see Figure 2.2), consisting of a touch screen monitor and a barcode scanner, which collected participant responses. A Tobii TX300 eye tracker was used to collect gaze behaviour while participants interacted with the simulated SCO. The eye tracker does not require goggles or head mounted gear; the participant merely sits in front of the screen while his/her eye movements are recorded. Participants were seated in front of the shopping item set-up. Although sitting at a SCO may not be considered ecologically valid, it was required to allow for eye tracking data to be collected in the sensitive zone in front of the screen. Item scanning movements were not impaired when participants were doing the task. 
There were 20 everyday shopping items used in the study. An example item was used for a demonstration in using the scanner and the touch screen monitor. Eighty-one screens were programmed using the eye-tracker software to represent the selfservice interface set-up based on a common selfservice checkout design.

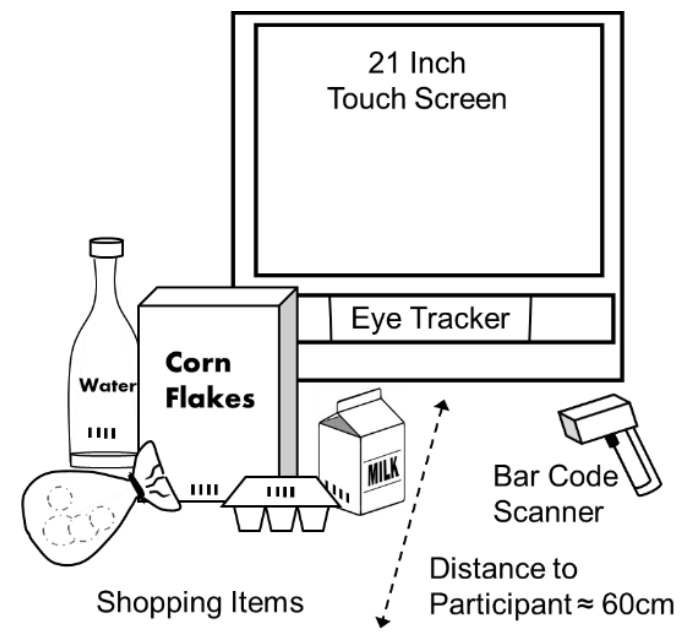

Figure 2.2: Set-up of the simulated SCO scenario. There were 20 shopping items in total (not shown here). The touch screen recorded participants' responses and the eye tracker cameras recorded participants' eye movements on the screen.

The agents were placed in the top right of the screen with the main SCO content screen being placed on the left, as users scan interfaces in a horizontal movement across the upper part of the content area (e.g. Djamasbi et al., 2011; Duggan et al. 2009; Pernice, 2017). The SCO content screen was also representative of a typical SCO interface to encourage a natural interaction (see Figure 2.3), with the touch screen recording participants' responses.

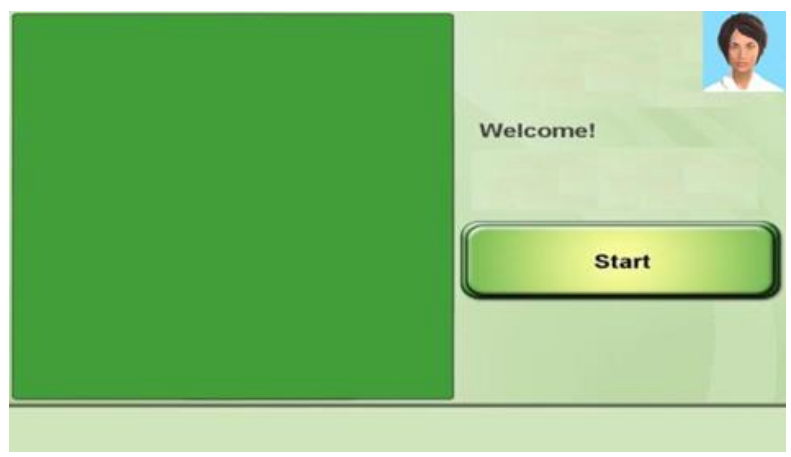

Figure 2.3: Example for the self-service checkout screens used in the study. Shown is the start screen of the scenario with the humanlike agent implementation.

\subsection{Procedure}

The experiment took place in a laboratory set-up at Abertay University. Upon arrival, all participants were given an information sheet providing details of the aim and procedure of the study, and an informed consent form in line with procedural requirements provided by the University's Ethics Committee. Initially, the information sheet withheld information from the participants by describing that the aim of the research was to examine the effectiveness of a new barcode reading system, to avoid biasing participants toward dis-/honest behaviour. It was emphasized that the study used an eye tracker as the gathered data would inform interface design. Participants were asked to sit on a seat in front of the eye tracker and shown an example of how to use the barcode scanner and the touch screen. Participants then completed a calibration process on the eyetracker and the researcher informed them that the eye tracker would merely record the eye position on the screen. Participants were also informed that they would complete the experiment alone in the room and that they were to go to a different room to tell the researcher when they were finished to reduce the social presence effects of the researcher.

The participant had to scan two shopping baskets, represented by two rows of items that were already laid out. Items were to be scanned in the order in which they were presented, and put to the right in a collection area after scanning. Participants were told that there were items that were to be scanned, weighed or counted. It was explained that items not provided with a bar code (i.e. that could not be scanned) were used simply to cause an interruption in the barcode reading process to resemble a reallife shopping experience. Unbeknownst to participants, items without a bar code provided opportunities for dishonest behaviours as participants were required to select a weight or provide item numbers.

In the first basket there were three items with attached weight labels. For each of the three items, participants had to select an item weight onscreen from three weight choices, each of which was different to the labelled weight shown on the respective item. Their choice of item weights represented the first opportunity of dishonesty, i.e. entering much lesser weights than were provided on the respective item weight labels. Participants were merely informed that there were a number of different items to test many barcodes and that the weights may not be accurate. If this were the case then participants were to select the weight they believed to be fair. This introduced the idea that the experimenter was unaware of the cost of the items.

The second basket provided three items to be counted, with the number for each item having to be entered, allowing for the second opportunity of dishonesty, i.e. entering smaller numbers than were provided for each item (see 2.3.1 for details on all opportunities for dishonesty, i.e. dilemmas).

Participants were asked to write down the total cost and how much change they were due after they had completed scanning each item row, as they would receive the change from $£ 10$ per basket, on a receipt slip. Thus, they would be receiving the 
change from £20 in total. This implied that the smaller the bill was, the more participants could financially benefit themselves. This was, however, not explicitly pointed out to participants. Participants were to give the receipt to the researcher at the end of the experiment by going into the adjacent room. Participants were told that the maximum change/payment they could get from taking part in the study would be up to $£ 5$.

A third opportunity for dishonesty was created by providing participants with the choice to incorrectly accept a money-off voucher after they had scanned all items (see 2.3.1).

Before participants began scanning the items, the researcher gave a quick demonstration of how to use the bar code scanner and touch screen. Participants then had the opportunity to practice the scanning process. After participants indicated they were comfortable with the task, the researcher left the participant alone in the room to complete the task.

At the end of the experiment, participants were fully debriefed on the true nature of the study, i.e. the aim of investigating dishonesty in participants, and were given the option to have their data removed. All participants agreed to their data being kept for analysis.

Participants signed an additional consent form at the end of the experiment to confirm given consent for their data to be used and to confirm that they had received their payment (i.e. the 'receipt' amount they reported to the experimenter).

\subsubsection{The dilemmas}

While scanning shopping items, participants were faced with three dilemmas that could result in undeserved financial gain. Three manipulations were used and adapted from Vohs and Schooler (2008) to expose participants to opportunities for dishonesty.

Dilemma 1: The first basket of shopping involved participants' receiving the change from $£ 10$. This amount had been pre-programmed to be $£ 2$, as the total bill of the items scanned equalled £8. Participants were instructed to scan items and select a weight shown on the screen for the products that had weights. A dilemma had been created as none of the weights were accurate and all of the weights that were presented were less than the stated weight on the shopping item. The participant had to choose from three slightly inaccurate weights presented on the screen. The weight buttons were $15 \mathrm{~g}, 30 \mathrm{~g}$ and $45 \mathrm{~g}$ less than the weight stated on the products. Each time the participant selected a weight that was greater than $15 \mathrm{~g}$ less, it was considered as acting opportunistically. In other words, the dilemma provided the participants with the possibility to act in an opportunistic manner by choosing a lesser weight than the one displayed on the product. In doing this, they may presume that the total cost of the basket would be reduced and that they may gain more money at the end of the experiment if they chose the lowest weight.

Dilemma 2: The second basket of shopping to be scanned also involved participants receiving the change from $£ 10$. This amount had been preprogrammed to be $£ 0.50$, as the total bill equalled $£ 9.50$. This basket involved participants scanning a row of items and selecting how many items there were of certain products, e.g., selecting the number of apples. Participants selected the number of items via a keypad on the screen. This trial measured whether participants chose to act in an opportunistic manner by choosing a lesser number of loose items (such as apples), as this may have been presumed to reduce the total cost of the basket.

Dilemma 3: At the end of the scanning process, participants were wrongly offered an opportunity to "accept" a $£ 1$ voucher as it claimed they had spent more than what the total came to. Participants could either accept or reject the voucher. Accepting the voucher indicated an instance of dishonest behaviour.

\subsection{Measures of analysis}

Dependent measures in relation to the hypotheses were:

Hypothesis $1(\mathrm{H} 1)$ :

- Average fixation duration

- Average fixation count

Both measures were retrieved from the area of interest containing the agents, which was fixed around the agent image. For each variable, data were pooled across basket scenarios.

Hypothesis $2(\mathrm{H} 2)$ :

- Receipt amounts

- Number of participants who accepted the voucher

- Instances of dishonest behaviours

Regarding the last measure, there were six opportunities to act opportunistically overall, i.e. three in each of the two basket scenarios. Data for receipt amounts and instances of dishonesty were pooled across basket scenarios respectively.

The three dependent measures were recorded in relation to $\mathrm{H} 2$, as we needed to explore which of these would be most sensitive to reveal potential for displaying dishonest behaviour, i.e. which measure was most diagnostic, avoiding potential ceiling or bottom effects. SPSS was used for statistical analysis.

Finally, and in order to gauge the extent to which the conditions were perceived to be different based on the agent condition, we ascertained whether 
there was a difference in perceived realism between the conditions compared to a real SCO setup. Participants rated their experience with the experimental set-up of a SCO compared to a real SCO experience on a scale of 1-3, from 1 being "not at all" like a SCO experience, 2 being "a little" and 3 being "a lot" like a SCO experience.

\section{RESULTS}

\subsection{H1: Fixation durations and counts}

Fixation durations and counts were not normally distributed, and thus were analysed using nonparametric tests. Kruskal-Wallis tests showed no significant differences across the three agent conditions, for either fixation durations, $X^{2}(2,39)=0.187$, $p>0.05$, or fixation counts, $X^{2}(2,38)=3.324, p>0.05$ (see Figure 3.1 for median fixation duration and count). Thus, Hypothesis 1 that a higher humanness level would result in greater attentional processing was not supported.

Visual inspection of participants' scan paths revealed that participants looked at the agents (see Figure 3.2). This suggests that the images of the agents were processed to some degree.

\subsection{H2: Receipt amounts, money vouchers falsely accepted and instances of dishonesty}

A Median test on the receipt amounts reported showed no significant difference between the agent conditions, $X^{2}(2,48)=1.5, p>0.05$.

A Chi-Square test indicated no significant effect of agent condition on the number of participants who accepted the money off voucher, $X^{2}(2,41)=0.927$, $p>0.05$, although there was a trend in the predicted direction. The highest number of acceptances occurred in the logo-like (16) condition, followed by the cartoon-like condition (14), with the humanlike condition resulting in the fewest acceptances (11).

A Median test on instances of dishonesty indicated a significant effect of agent humanness condition, $X^{2}(2,48)=7.371, p<0.05$, with a moderate-large effect size, $\varphi_{c}=0.392$ (see Figure 3.3 for counts of instances of dishonesty). Post-hoc tests revealed a significant difference between the logo-like and humanlike agent condition, $X^{2}(1,32)=6.149$, $p<0.017$ (with Bonferroni correction); the difference between the logo-like vs cartoon-like condition missed significance with Bonferroni correction, $p>0.017,\left(X^{2}(1,32)=4.5, p=0.034\right)$. The comparison between the cartoon-like and human-like agent showed no significant effect ( $p>0.017)$.

Thus, Hypothesis 2 that a higher humanness level (higher social presence) resulted in less dishonest behaviour to a lower humanness level (lower social presence) was only partially supported.

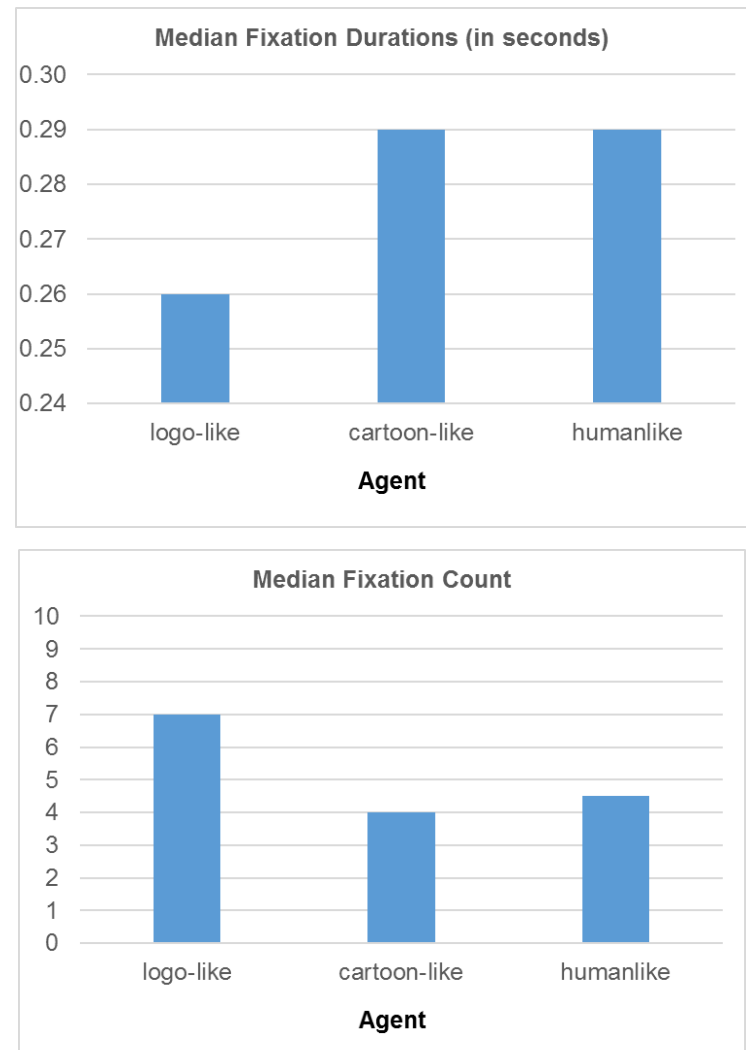

Figure 3.1: Median fixation duration (in seconds) and median fixation count for the agent conditions.
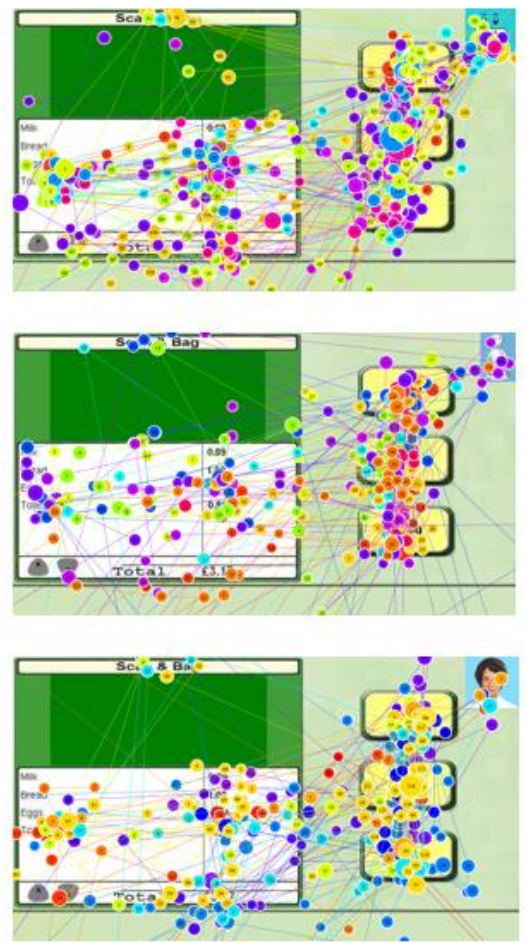

Figure 3.2: An example of participants' scan paths across the checkout screen for logo-like agent (top panel), cartoon-like agent (middle panel), and humanlike agent (bottom panel). Each differently coloured dot represents the scan path of a different participant (note: dots may overlap and may thus not be visible). 


\subsection{Realism}

A Median test to determine whether there was a difference between the agent conditions compared to a real SCO set-up showed significant differences for the realism ratings, $X^{2}(2,48)=6.5, p<0.05$, with a moderate-large effect size, $\varphi_{c}=0.368$. Median ratings were 3 ("A lot like a SCO experience") for the cartoon-like and humanlike agent condition, and 2 ("A little like a SCO experience") for the logo-like agent condition. Post-hoc tests showed a significant difference between the logo-like and humanlike condition, $\mathrm{X}^{2}(2,32)=6.149, \mathrm{p}<0.017$ (with Bonferroni correction), in terms of realism ratings. All other comparisons were not significant, all $p>0.017$.

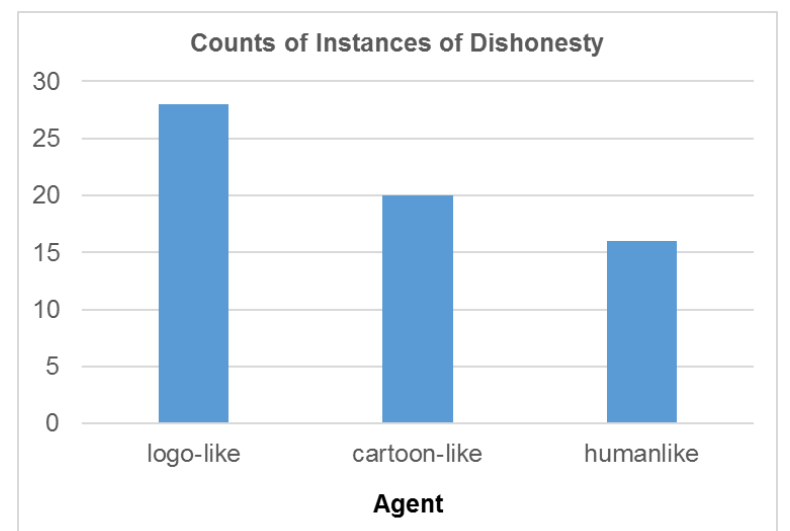

Figure 3.3: Counts of instances of dishonesty in the agent conditions ( $n=16$ in each agent condition).

\section{DISCUSSION}

The present study investigated the influence of social presence, instantiated by different levels of humanness of interface agents, on opportunistic behaviour and attention distribution.

It was predicted that a high humanness level $(\mathrm{HA})$, attracted more user attention $(\mathrm{H} 1)$ and reduced opportunistic behaviour (H2), compared to a low humanness condition (CA and LA).

With respect to the first Hypothesis, the results showed that fixation durations were not significantly different across agent conditions. As fixation duration can be an index of attention (Duchowski, 2002), it appears all agents equally attracted attention, and the humanlike agent did not receive longer fixations than any of the others. There are several possible reasons for this finding. First, it should be noted that in all agent conditions, participants were merely instructed to scan the items, in line with a shopping transaction; the presence of an agent could be deemed irrelevant by participants. Gaze behaviour is usually determined by the task a user engages with (see Duchowski, 2002), and it may well be the case that participants found the agents irrelevant to the task in hand. Related to that, and for reasons of ecological validity, the focus of the task was to engage in a SCO transac- tion, and participants were instructed accordingly. Others have reported that an on-screen, visual humanlike agent in a simulated SCO transaction resulted in fewer participants making errors compared to a control condition, although participants did not consciously acknowledge the presence of the visual agent (Payne et al., 2011). While there is limited or mixed evidence that human faces are processed outside consciousness, this may not be the case for unfamiliar faces (for a review see Axelrod, 2015). There is also evidence that faces can be processed automatically (Pessoa, 2005; Richler et al., 2011), so perhaps the humanlike agents could have had an effect on a less observable level than could be revealed by eye tracking, which is consistent with Payne et al. (2011). However, the findings are still inconsistent with Hypothesis 1, and Devue et al.'s (2009) research that human faces receive more attention than objects.

In relation to the average fixation count, there were no significant differences between the different agent conditions, though participants appeared to look more often at the logo-like agent compared to the other agents. Higher fixation frequency is typically associated with importance of a viewed area. However, given that the logo-like agent contained a small area of text, the findings may have been due to the lack of complexity in the cartoon-like agent, especially given that the cartoon-like agent looked rather simple, however, still resembled a face. The trend in the findings may suggest that the logo-like agent was simply 'different' to the other agents as it contained textual information. The processing of the cartoon- and humanlike agent was perhaps less resource intense, in that an image once classified as a face may be dismissed easily, especially if faces are processed automatically. The text in the logo-like agent may have prompted participants to read it, resulting in more fixations as participants scanned the words. Future studies should aim to control the information content in the agent images to account for this factor. However, we can be reasonably confident based on the visual inspection of participants' gaze behaviour that the agents were looked at and thus provided an opportunity to be processed to some level, which was required for ascertaining social presence effects.

The second Hypothesis suggested that higher levels of humanness (social presence), would result in less dishonest participant behaviour, and this predication was partially supported.

There was no significant effect of agent condition on the amount on the receipts returned, so this finding is not consistent with Hypothesis 2. We can speculate that the manipulation was perhaps not sufficiently large to be of consequence, given that the extent to which participants could benefit themselves was small. If participants were totally honest they would report being owed $£ 2.50$ (minimum 
participant payment) and if they were maximally dishonest they could additionally claim up to $£ 1.50$ across both basket scenarios. Thus, the manipulation was perhaps less effective in that it was not sensitive enough to flag up large differences between agent conditions.

Most participants in all agent conditions accepted and reported to the researcher that they were due the $£ 1$ voucher that was wrongfully suggested to be owed to them, which is also inconsistent with our expectations. This finding may suggest that people are generally inclined to want to maximise their gain, and that social presence had no effect. Another explanation may be that a ceiling effect may have occurred, because participants had only two choices (accept or reject voucher), leaving little space for differentiation. However, the finding may also suggest that participants were confused by the statement that said "As you have spent over $£ 10$ you are due a $£ 1$ off voucher". Participants may not have realised that accepting the voucher that was wrongly proposed was a form of dishonesty. They may have interpreted that the system suggested that they had indeed spent more than $£ 10$. Thus, this measure may not be an effective or clear way to assess dishonesty. The method used within Vohs and Schooler's (2008) research, where participants were to press the space bar to avoid dishonest behaviour may provide a more effective design for interpreting levels of dishonest behaviour. Alternatively, a rephrasing of the statement used in the study to "If you have spent over $£ 10$ you can accept the voucher" may have been a clearer instruction. A default procedure whereby participants actively have to avoid dishonesty may perhaps be a more refined experimental manipulation.

However, the third dependent measure, instances of dishonesty, was affected by the humanness of the agent, with the humanlike agent being associated with significantly fewer instances of dishonesty than the lowest instantiation of social presence via the logo-like agent. This finding provided evidence that social presence can affect dis-/honest customer behaviour, consistent with Hypothesis 2. The effect, while significant, may be seen as small, with effectively one instance of theft per customer fewer for the humanlike agent compared to the least humanlike agent condition in practical terms. However, if only one fewer item is stolen per customer transaction in retail, then this would result in substantial savings for businesses. This finding thus supports the notion that social presence in more humanlike agent format may reduce the occurrence of dishonest behaviour, at least in some instances, and is consistent with the literature that associates prosocial behaviours with social presence (Bateson et al., 2006; Parise, 1999).

For studies of this type to be informative for interface designers and businesses, it needs to be con- sidered how well the findings transfer to a real-life application. In this study, the cartoon-like and humanlike agent conditions were perceived to be sufficiently realistic compared to a typical SCO environment, so we can be reasonably confident that there is some degree of ecological validity. The logo-like agent was perceived to be less realistic, which may limit the generalisability of findings.

It should be noted that the study gave participants the choice to act in a dishonest manner, which in itself raises ethical issues. Investigating issues of this nature is rather challenging. However, given the continued problem of shrinkage in retail, and the increase of technological implementation that may reduce social presence, it seems vital that studies explore the role of measures that can reduce theft.

It is important to highlight that the use of SCOs within a store setting is subject to many other factors that may affect opportunistic behaviour, such as the number of SCOs, the number of customers at the SCOs, busyness of the store, layout, etc. (Creighton et al., 2015). Thus, the generalizability of the findings may be limited in this respect. Future research may want to explore whether these factors have an effect on opportunistic behaviour, and how these are mediated by social presence.

\subsection{Conclusions}

To conclude, the study has partially demonstrated that social presence had an effect on opportunistic behaviour using visual agents, in that instances of dishonest behaviour were reduced with the humanlike agent. However, the humanlike agent did not draw more attention compared to the cartoon-like or logo-like agent conditions. The findings overall are mixed, and more research should be conducted into the effects of instantiating social presence, and measures to explore dishonesty.

We suggest that there is scope to investigate social presence effects on dis-/honest consumer behaviour, given the substantial evidence for social presence to induce prosocial behaviours. Designers of interfaces interested in this field need to achieve a balance in that an agent will have to be noticed sufficiently, while not interfering with a consumer's task in a SCO transaction.

Research in this area may be of particular interest to companies who increasingly put the customer at the centre of providing their own service, where shopping may become more or totally independent from staff interaction, as, for example, provided by Amazon Go, introducing the first checkout free store (Amazon, 2018). The study of social presence effects on customer behaviour may thus become all the more important as the landscape for consumer interactions changes. Other researchers have already suggested that social el- 
ements, and thereby social presence, in retail will become more and more relevant for customer service experiences (Van Doorn et al., 2017). Given the increasing use of technology that makes the customer self-sufficient for retail transactions, as well as the rapid development of Artificial Intelligence substituting staff activities, there is potentially huge merit in maintaining a social element in consumer interaction with technology via social presence, and this may work for the benefit of businesses and consumers.

\section{REFERENCES}

Amazon (2018) Amazon Go. Available from: https://www.amazon.com/b?ie=UTF8\&node $=160$ 08589011 [accessed 9 April 2018].

Antes, J.R. (1974) The time course of picture viewing. Journal of experimental psychology, 103 (1), 62.

Axelrod, V., Bar, M. and Rees, G. (2015) Exploring the unconscious using faces. Trends in cognitive sciences, 19 (1), 35-45.

Bamfield, J. (2004) Shrinkage, shoplifting and the cost of retail crime in Europe: a cross-sectional analysis of major retailers in 16 European countries. International journal of retail \& distribution management, 32 (5), 235-241.

Baron-Cohen, S. (1997) How to build a baby that can read minds: Cognitive mechanisms in mindreading. The maladapted mind: Classic readings in evolutionary psychopathology, 207-239.

Bateson, M., Nettle, D. and Roberts, G. (2006) Cues of being watched enhance cooperation in a real-world setting. Biology letters, 2 (3), 412-414.

Baumeister, R.F. (1982) A self-presentational view of social phenomena. Psychological bulletin, 91, 3-26.

Baumer, T. and Rosenbaum, D. (1984) Combating retail theft: Programs and strategies. Stoneham, MA: Butterworth.

Beck, A. (2011) Self-scan checkouts and retail loss: Understanding the risk and minimising the threat. Security journal, 24 (3), 199-215.

Beck, A. (2015) Developments in retail mobile scanning technologies: Understanding the potential impact on shrinkage \& loss Prevention. Leicester: ESRC.

Beck, A. and Hopkins, M. (2017) Scan and rob! Convenience shopping, crime opportunity and corporate social responsibility in a mobile world. Security journal, 30 (4), 1080-1096.

Beck, A. and Willis, A. (1999) Context-specific measures of CCTV effectiveness in the retail sector. Surveillance of public space: CCTV, street lighting and crime prevention. Crime prevention studies series, 10, 251-269.

Biocca, F., Harms, C. and Gregg, J. (2001) The Networked Minds measure of social presence. Pilot test of the factor structure and concurrent validity. Paper presented at the 4th Annual International Workshop on Presence 2001. Philadelphia, USA.

Biocca, F., Harms, C. and Burgoon, J.K. (2003) Towards a more robust theory and measure of social presence: Reviews and suggested criteria. Presence, 12 (5), 456-480.

Carmel-Gilfilen, C. (2013) Bridging security and good design: Understanding perceptions of expert and novice shoplifters. Security journal, 26 (1), 80-105.

Clarke, R. V. and Cornish, D. B. (1985) Modeling offenders' decisions: A framework for research and policy. Crime and justice, 6, 147-185.

Clarke, R.V. and Eck, J.E. (2014) Become a problem-solving crime analyst. London: Routledge.

Cornish, D.B. and Clarke, R.V. (1987) Understanding crime displacement: An application of rational choice theory. Criminology, 25 (4), 933-948.

Creighton, S., Johnson, G., Robertson, P., Law, J. and Szymkowiak, A. (2015) Dishonest behavior at self-service checkouts. In: International Conference on $\mathrm{HCl}$ in Business. Champ.: Springer, 267-278.

Cyr, D., Hassanein, K., Head, M. and Ivanov, A. (2007) The role of social presence in establishing loyalty in e-service environments. Interacting with computers, 19 (1), 43-56.

Dautenhahn, K. and Billard, A. (2002) Games children with autism can play with Robota, a humanoid robotic doll. In: Universal access and assistive technology. London: Springer, 179-190.

Devue, C., Laloyaux, C., Feyers, D., Theeuwes, J. and Brédart, S. (2009) Do pictures of faces, and which ones, capture attention in the inattentional blindness paradigm? Perception, 38 (4), 552568.

Djamasbi, S., Siegel, M., skorinko, J. and Tullis, T. (2011) Online viewing and aesthetic preferences of generation $\mathrm{Y}$ and the baby boom generation; testing user web site experience through eye tracking. International journal of electronic commerce,15 (4), 121-157.

Duchowski, A.T. (2002) A Breadth-First Survey of Eye Tracking Applications. Behaviour Research Methods, Instruments and computers, 34 (4), 455-470.

Duggan, G.B. and Payne, S.J. (2009) Text skimming: The process and effectiveness of foraging 
through text under time pressure. Journal of experimental psychology: Applied, 15 (3), 228-242.

Emery, N.J. (2000) The eyes have it: the neuroethology, function and evolution of social gaze. Neuroscience \& biobehavioral reviews, 24 (6), 581-604.

Friedman, A. and Liebelt, L.S. (1981) On the time course of viewing pictures with a view towards remembering. Eye movements: Cognition and visual perception, 137-155.

Gefen, D. and Straub, D.W. (2004) Consumer trust in B2C e-Commerce and the importance of Social Presence: Experiments in e-Products and eServices. The international journal of management science, 32 (6), 407-422.

Haxby, J.V., Hoffman, E.A. and Gobbini, M.I. (2000) The distributed human neural system for face perception. Trends in cognitive sciences, 4 (6), 223-233.

Henderson, J.M. (2007) Regarding scenes. Current directions in psychological science, 16 (4), 219222.

Home Office (2015) Crime against businesses: Findings from the 2014 commercial victimisation survey. London: Home Office. Available from: https://www.gov.uk/government/publications/crim e-against-businesses-findings-from-the-2014commercial-victimisation-survey/crimes-againstbusinesses-findings-2014 [accessed 9 April 2018].

Kajalo, S. and Lindblom, A. (2010) The perceived effectiveness of surveillance in reducing crime at shopping centers in Finland. Property management, 28 (1), 47-59.

King, W.J. and Ohya, J. (1996) The representation of agents: Anthropomorphism, agency, and intelligence. In: Conference Companion on Human Factors in Computing Systems (CHI'96). New York, USA: ACM, 289-290.

Klemke, L.W. (1982) Exploring adolescent shoplifting. Sociology and social research, 67, 59-75.

Koda, T. and Maes, P. (1996) Agents with faces: The effect of personification. In: Robot and Human Communication, 1996, 5th IEEE International Workshop. IEEE, 189-194.

Kraut, R.E. (1976) Deterrent and definitional influences on shoplifting. Social problems, 25, 358368.

Küster, D., Krumhuber, E. and Kappas, A. (2015) Nonverbal behavior online: A focus on interactions with and via artificial agents and avatars. In: The social psychology of nonverbal communication. UK: Palgrave Macmillan, 272-302.
Lee, M., Kim, K., Daher, S., Raij, A., Schubert, R., Bailenson, J. and Welch, G. (2016) The wobbly table: Increased social presence via subtle incidental movement of a real-virtual table. In: Virtual Reality (VR), 2016 IEEE. IEEE. 11-17.

Martinez, S., Sloan, R. J., Szymkowiak, A. and Scott-Brown, K. C. (2011) Animated virtual agents to cue user attention: Comparison of static and dynamic deictic cues on gaze and touch responses. International journal on advances in intelligent systems. 4 (3 \& 4), 299-308.

Nowak, K. L. and Biocca, F. (2003) The effect of the agency and anthropomorphism on users' sense of telepresence, co-presence, and social presence in virtual environments. Presence, 12 (5), 481-494.

Parise, S., Kiesler, S., Sproull, L. and Waters, K. (1999) Cooperating with life-like interface agents. Computers in human behavior, 15 (2), 123-142.

Pernice, K. (2017) F-shaped pattern of reading on the web: Misunderstood, but still relevant (even on mobile). Nielsen Norman Group, 12 November 2017. Available from: https://www.nngroup.com/articles/f-shapedpattern-reading-web-content//accessed 4 April 2018].

Payne, J.A., Johnson, G.I. and Szymkowiak, A. (2011) The behavioural impact of a visually represented virtual assistant in a self-service checkout context. In: Proceedings of the 25th BCS Conference on Human-Computer Interaction. Newcastle-upon-Tyne, UK: British Computer Society, 58-63.

Pessoa, L. (2005) To what extent are emotional visual stimuli processed without attention and awareness? Current opinion in neurobiology, 15 (2), 188-196.

Peters, C. (2005) Direction of attention perception for conversation initiation in virtual environments. In: International Workshop on Intelligent Virtual Agents. Berlin, Heidelberg: Springer, 215-228.

Pfattheicher, S. and Keller, J. (2015) The watching eyes phenomenon: The role of a sense of being seen and public self-awareness. European journal of social psychology, 45 (5), 560-566.

Richler, J.J., Wong, Y.K. and Gauthier, I. (2011) Perceptual expertise as a shift from strategic interference to automatic holistic processing. Current directions in psychological science, 20 (2), 129-134.

Romano, D.M., Sheppard, G., Hall, J., Miller, A. and Ma, Z. (2005) BASIC: A Believable, Adaptable Socially Intelligent Character for Social Presence. PRESENCE 2005, The 8th Annual International Workshop on Presence, 21-22 
September 2005, University College London, London, UK.

Shinozawa, K., Naya, F., Yamato, J. and Kogure, K. (2005) Differences in effect of robot and screen agent recommendations on human decision-making. International journal of humancomputer studies, 62 (2), 267-279.

Short J., Williams E. and Christie B. (1976) The Social psychology of telecommunications. London: John Wiley \& Sons.

Siebenaler, S., Szymkowiak, A., Robertson, P., Johnson, G. and Law, J. (2017) Social Presence and Dishonesty: Perceptions from Security Guards. In: International Conference on $\mathrm{HCl}$ in Business, Government, and Organizations. Champ: Springer, 264-281.

Sproull, L., Subramani, M., Kiesler, S., Walker, J.H. and Waters, K. (1996) When the interface is a face. Human-computer interaction, 11 (2), 97124.

Taylor, E. (2016) Supermarket self-checkouts and retail theft: The curious case of the SWIPERS. Criminology \& criminal justice, 16 (5), 552-567.

Unema, P.J., Pannasch, S., Joos, M. and Velichkovsky, B.M. (2005) Time course of information processing during scene perception: The relationship between saccade amplitude and fixation duration. Visual cognition, 12 (3), 473-494.

Van Doorn, J., Mende, M., Noble, S.M., Hulland, J., Ostrom, A.L., Grewal, D. and Petersen, J.A., (2017) Domo arigato Mr. Roboto: Emergence of automated social presence in organizational frontlines and customers' service experiences. Journal of service research, 20 (1), 43-58.

Vohs, K. D. and Schooler, J. W. (2008) The value of believing in free will: Encouraging a belief in determinism increases cheating. Psychological science, 19 (1), 49-54.

Walther, J.B. (1992) Interpersonal effects in computer-mediated interaction a relational perspective. Communication research, 19 (1), 52-90.

Welsh, B.C., Farrington, D.P. and O'Dell, S.J. (2010) Effectiveness of public areas surveillance for crime prevention: Security guards, place managers and defensible space. Stockholm: Swedish National Council for Crime Prevention.

Wirtz, J. and McColl-Kennedy, J.R. (2010) Opportunistic customer claiming during service recovery. Journal of the academy of marketing science, 38 (5), 654-675.

Zhao, S. (2003) Toward a taxonomy of copresence. Presence, 12 (5), 445-455. 